\title{
Fluctuation-dissipation relation from the nonequilibrium dynamics of a nonlinear open quantum system
}

\author{
Jen-Tsung Hsiang $\odot^{*}$ \\ Center for High Energy and High Field Physics, National Central University, Chungli 32001, Taiwan \\ and Center for Particle Physics and Field Theory, Department of Physics, \\ Fudan University, Shanghai 200438, China \\ Bei-Lok $\mathrm{Hu}$ \\ Maryland Center for Fundamental Physics and Joint Quantum Institute, \\ University of Maryland, College Park, Maryland 20742, USA
}

(Received 23 February 2020; accepted 30 April 2020; published 2 June 2020)

\begin{abstract}
Continuing our inquiry into the conditions when fluctuation-dissipation relations (FDR) may appear in the context of nonequilibrium dynamics of open quantum systems (over and beyond the conventional FDR from linear response theory) we turn to non-Gaussian systems and consider this issue for an anharmonic quantum oscillator interacting with a scalar quantum field bath. We present the general nonperturbative expressions for the rate of energy (power) exchange between the anharmonic oscillator and its thermal bath. For the cases that a stable final equilibrium state exists, and the nonstationary components of the two-point functions of the anharmonic oscillator have negligible contributions to the power balance, we can show nonperturbatively that equilibration implies an FDR for the anharmonic oscillator. We then use a weakly anharmonic oscillator as an example to illustrate the validity of those two assumptions and show that in the weak anhamonicity limit, they are satisfied according to our first-order perturbative results..
\end{abstract}

DOI: 10.1103/PhysRevD.101.125003

\section{INTRODUCTION}

The open system paradigm captures physical reality better than the idealization of a system in total isolation because the environment it interacts often plays a role. The interlocking relation between the open system and its environment is registered in the fluctuation-dissipation relations (FDRs). While they are rooted in statistical mechanics [1-5] the implications of FDRs are wide ranging, from condensed matter [6,7], nuclear/particle (e.g., [6,8]) to black hole physics (e.g., [9,10]) and cosmology (e.g., [11]). Further description of its scope can be found in $[1,12,13]$.

In this paper we investigate the FDR for a nonlinear quantum system interacting linearly with a thermal bath: the system of interest is a quantum anharmonic oscillator and the bath is made up of a thermal scalar quantum field. Two primary aspects in statistical mechanics are involved here for both classical and quantum systems: A. FDR in the

\footnotetext{
*cosmology@gmail.com

†blhu@umd.edu
}

Published by the American Physical Society under the terms of the Creative Commons Attribution 4.0 International license. Further distribution of this work must maintain attribution to the author(s) and the published article's title, journal citation, and DOI. Funded by SCOAP. context of 1) response theory versus 2) nonequilibrium dynamics, B. FDR for 1) linear vs 2) nonlinear systems. The case of FDR in linear response theory (LRT) - linear systems treated in response theory $(\mathrm{A} 1+\mathrm{B} 1)$ is well known from standard textbooks and discussed most widely. For nonlinear systems treated by response theory $(\mathrm{A} 1+\mathrm{B} 2)$ both in the near-equilibrium and nonequilibrium contexts, there is also a long history of significant theoretical development by authors like Kadanoff and Baym [6], Eremov [14], Golden et al. [15], Zwangzig [16], Langreth [17], Zhou et al. [18], Bochkov and Kuzovlev [19] and Stratonovich [20]. In recent work, Wang and Heniz [21] derive a nonlinear generalization of the fluctuation-dissipation theorem (for the $n$-point Green's functions and the amputated one-particle irreducible vertex functions) at finite temperature. (See also Carrington et al. [22].) The methods used in the work of Miyazaki and Reichman [23], Maciejko et al. [24], and Motz et al. [25] are also of interest, so is the more recent out-of-time-order fluctuation-dissipation relation of Ueda's group [26].

Before we mention some more recent developments, for clarification purpose, it is perhaps useful to highlight the differences (feature A above) in the formulation of FDR between the nonequilibrium dynamics (NEq) approach which we follow in our work and the conventional linear (LRT) or nonlinear response theory. 


\section{A. FDR in LRT vs NEq}

The main differences in the setup, the conditions and the features have been described in Sec. 3 of [27]. We summarize them as follows:

(i) Setup: FDR under LRT operates under the following assumptions: the system of interest (i) has been prepared in a thermal state in thermal equilibrium with the bath, and then (ii) is taken away by a weak external disturbance and the corresponding responses is recorded. By comparison, in the nonequilibrium (NEq) formalism, the system can start in any state, which may be very different from the thermal state at the bath temperature or the equilibrium state the system finally settles in. Once the initial state of the system and the properties of the bath are given, their interaction determines the evolution history described by the reduced dynamics of the system. If the dynamics of the system comes to equilibration, then an FDR will be determined for that equilibrium state.

(ii) Conditions: In LRT the equilibrium state of the system which is assumed to hold for all times. The FDR in LRT is formulated with respect to this equilibrium state in terms of perturbation theoryweak coupling with the bath and small perturbation from the equilibrium state. In nonlinear response, nonlinear perturbance is considered, but response functions are well defined and calculated assuming that the perturbance does not exceed a certain limit. In NEq context, the system and its environment can be strongly coupled while dynamically evolving, but the existence of an equilibrium state is not a priori known. One needs to first examine if the system will relax to an equilibrium state before one attempts to verify that an FDR exists. Thus FDR in NEq context is an emergent phenomenon depending on many factors which enter into determining the dynamics of the open system.

(iii) Features: In the NEq dynamics approach the equilibrium state which an open system evolves to in general is not a Gibbs (thermal) state. The temperature is ill-defined in a dynamical setting only if/ until the system reaches equilibrium, whence it can be identified from the reduced density matrix or the physical observables of the equilibrated system. Even so it is at best an "effective" temperature because it depends on the details of the system and the bath parameters. The exceptional situation is when the coupling strength between the system and the bath is vanishingly small, which is a preamble of LRT. Thus in the context of NEq formulation, the temperature appearing in the proportionality constant of the FDR is in fact the initial temperature of the bath, not the temperature of the system in the final equilibrium state, in contrast to LRT.
In essence, the FDR in LRT plays a spectator role, connecting the response of the system to an external disturbance. In comparison, in the $\mathrm{NEq}$ formulation, FDR has a dynamical significance since it ensures the balance of the energy flow between the reduced system and the environment [27], which in turn signifies dynamical relaxation of the system into equilibrium.

\section{B. This work: FDR from the nonequilibrium dynamics of non-Gaussian quantum systems}

In our recent work we have explored the nonequilibrium dynamics and the FDR in two settings: A) A system of interacting quantum harmonic oscillators sharing a common thermal bath of quantum scalar field and their FDR at late times [4,13], B) a chain of harmonic oscillators with terminals connected to two baths of different temperatures in a nonequilibrium steady state [5]. In both cases the system-bath setup being Gaussian enables us to produce exact formal solutions for the dynamics. In the preceding paper [28], we take a baby step toward non-Gaussian systems, ${ }^{1}$ in treating nonlinear quantum systems with the help of perturbative methods. In this work, we consider the existence of an FDR for an anharmonic quantum oscillator interacting with a scalar quantum field bath. We use the functional method originated in [30] and developed for this problem in [28] to obtain general, nonperturbative expressions for the rate of energy (power) exchange between the anharmonic oscillator and the thermal bath. Under the assumptions that a stable unique final equilibrium state exists, and the nonstationary components of the two-point functions of the anharmonic oscillator have negligible contributions to the evaluation of the power balance, we can demonstrate nonperturbatively that equilibration implies an FDR for the anharmonic oscillator. This result establishes a possible connection between equilibration and the FDR in a more general setting. It also shows that the nontrivial $n$-point functions, with $n>2$, of this nonGaussian system do not play any explicit role in the derivation of the FDR.

We then use a weakly anharmonic oscillator as an example to illustrate that these two assumptions indeed are satisfied according to our first-order perturbative results in the configuration we chose: that the net energy exchange vanishes after relaxation in the open system dynamics, and an equilibrium state exists at late times. We point out several implications of the perturbative results and call attention to the roles the environment plays in the dynamics of the nonlinear system.

This paper is organized as follows. In Sec II, we briefly summarize the essence of the functional method adopted here for the problem of a quantum anharmonic oscillator

\footnotetext{
${ }^{1}$ Non-Gaussian systems are wide-ranged and their behavior can be very different, e.g., mixing and ergodicity can be violated and there is no guarantee of an FDR [29].
} 
coupled to a thermal field, and highlight the results [28] regarding the late-time behaviors of the Green's functions, in particular, the retarded Green's function and the Hadamard function, of the oscillator to the first order in the anharmonic potential of the form $\frac{\lambda}{4 !} \chi^{4}$ with $\lambda>0$. In Sec. III, we formally derive a nonperturbative expression for the net energy exchange between the anharmonic oscillator and the bath field, and argue that based on the prerequisite assumptions, the balance of this energy flow can imply a nonperturbative FDR for the anharmonic oscillator after its dynamics is relaxed to the final equilibrium state. Finally, in Sec. IV, we apply the functional perturbative approach to a weakly anharmonic oscillator and show that to first order in the anharmonicity, the assumptions adopted in Sec. III hold naturally, and discuss the pivotal roles the environment plays in the dynamics of the anharmonic oscillator.

\section{NONEQUILIBRIUM EVOLUTION OF A DRIVEN ANHARMONIC OSCILLATOR IN A THERMAL BATH}

\section{A. In-in generating functional [31]}

We consider an anharmonic oscillator driven by an external current $j$. It has mass $m$ and bare natural frequency $\omega_{0}>0$, and is coupled with arbitrary strength $e$ to a bath of massless quantum scalar field $\phi(x)$, initially (at $t=0$ ) prepared in a thermal state. The corresponding action is given by

$$
\begin{aligned}
S_{V}[\chi, \phi] & =\int_{0}^{t} d s\left\{\frac{m}{2}\left[\dot{\chi}^{2}(s)-\omega_{0}^{2} \chi^{2}(s)\right]-V[\chi(s)]+j(s) \chi(s)\right\} \\
& +\int_{0}^{t} d^{4} x e \chi(s) \delta^{3}(\mathbf{x}-\mathbf{z}(s)) \phi(x) \\
& \left.+\int_{0}^{t} d^{4} x \frac{1}{2}\left[\partial_{\mu} \phi(x)\right]\left[\partial^{\mu} \phi(x)\right]\right\}
\end{aligned}
$$

The anharmonic potential $V$ will be chosen to be a monomial in the displacement $\chi$ of the oscillator, although the functional formalism we adopt here is not restricted to this condition. The self-coupling constant $\lambda$ associated with the potential will be assumed to be sufficiently weak if we treat it as a perturbation. The parameter $\mathbf{z}$ denotes the location of the oscillator. Thus Eq. (2.1) describes the case of an anharmonic oscillator in the dipole approximation, or equivalently, an Unruh-DeWitt detector, whose internal degree of freedoms are now modeled by an anharmonic oscillator.

In [28], we have shown that given the initial state of the oscillator at $t=0$, the reduced density matrix $\rho_{\chi}^{(V)}$ of the anharmonic oscillator at a later time $t$ is given by

$$
\begin{aligned}
\rho_{\chi}^{(V)}\left(q_{b}, r_{b}, t\right)= & \exp \left\{-i \int_{0}^{t} d s\left[V\left(\frac{\delta}{i \delta j_{+}}\right)-V\left(-\frac{\delta}{i \delta j_{-}}\right)\right]\right\} \\
& \times\left.\rho_{\chi}\left(q_{b}, r_{b}, t\right)\right|_{j_{q}=0=j_{r}}
\end{aligned}
$$

where $\rho_{\chi}\left(q_{b}, r_{b}, t\right)$ is the reduced density matrix element of the free oscillator driven by the source $j_{ \pm}$, and

$$
\begin{gathered}
q=\frac{\chi_{+}+\chi_{-}}{2}, \quad \frac{\delta}{\delta j_{+}}=+\frac{\delta}{\delta j_{q}}+\frac{1}{2} \frac{\delta}{\delta j_{r}}, \\
r=\chi_{+}-\chi_{-}, \quad \frac{\delta}{\delta j_{-}}=-\frac{\delta}{\delta j_{q}}+\frac{1}{2} \frac{\delta}{\delta j_{r}} .
\end{gathered}
$$

Thus the expectation value of an operator $\mathcal{O}$ consisting only of the anharmonic oscillator variables is given by

$$
\begin{aligned}
\langle\mathcal{O}(t)\rangle= & \frac{1}{\mathcal{Z}_{V}} \operatorname{Tr}\left\{\mathcal{O} \rho_{\chi}^{(V)}(t)\right\} \\
= & \frac{1}{\mathcal{Z}_{V}} \exp \left\{-i \int_{0}^{t} d s\left[V\left(\frac{\delta}{i \delta j_{+}}\right)-V\left(-\frac{\delta}{i \delta j_{-}}\right)\right]\right\} \\
& \times\left.\langle\hat{\mathcal{O}}\rangle^{(0)} \mathcal{Z}\right|_{j_{q}=0=j_{r}}
\end{aligned}
$$

with $\mathcal{Z}=\operatorname{Tr}\left\{\rho_{\chi}(t)\right\}, \mathcal{Z}_{V}=\operatorname{Tr}\left\{\rho_{\chi}^{(V)}(t)\right\}$ being the "in-in" generating functional of the free oscillator and the anharmonic oscillator respectively at the final time $t$ to ensure proper normalization. The superscript 0 on an expectation value $\langle\hat{\mathcal{O}}\rangle^{(0)}$ refers to the results without the anharmonic potential.

The generating functional $\mathcal{Z}$ has been shown [28] to take on the form,

$$
\begin{aligned}
\mathcal{Z}\left[j_{q}, j_{r}\right]= & \exp \left\{-\frac{1}{2} \int_{0}^{t} d s \int_{0}^{t} d s^{\prime} j_{q}(s) G_{H, 0}^{(\chi)}\left(s, s^{\prime}\right) j_{q}\left(s^{\prime}\right)\right. \\
& \left.+i \int_{0}^{t} d s \int_{0}^{s} d s^{\prime} j_{q}(s) G_{R, 0}^{(\chi)}\left(s-s^{\prime}\right) j_{r}\left(s^{\prime}\right)\right\},
\end{aligned}
$$

for any Gaussian initial state, where $G_{H, 0}^{(\chi)}\left(s, s^{\prime}\right)$ and $G_{R, 0}^{(\chi)}\left(s-s^{\prime}\right)$ are respectively the noise kernel and the dissipation kernel of the linear oscillator coupled with the bath field initially at temperature $\beta^{-1}$. In the language of perturbative treatments, the linear or harmonic case is at the "zeroth-order" of anharmonicity. The full generating functional $\mathcal{Z}_{V}$ in the presence of a nonlinear potential $V$ can be expanded with respect to the small self-coupling in the nonlinear potential into the form,

$$
\begin{aligned}
\mathcal{Z}_{V} & =\exp \left\{-i \int_{0}^{t} d s\left[V\left(\frac{\delta}{i \delta j_{+}}\right)-V\left(-\frac{\delta}{i \delta j_{-}}\right)\right]\right\} \mathcal{Z} \\
& =\mathcal{Z}+\mathcal{Z}_{1}+\cdots
\end{aligned}
$$

where $\mathcal{Z}_{1}$ is the leading order correction of $\mathcal{Z}$ due to the nonlinear potential $V$, assuming the nonlinearity is relatively weak. It has been shown [28] that in the limit $j \rightarrow 0$, there is no first-order correction of $\mathcal{Z}_{V}$ due to the nonlinear potential. 
Equation (2.5) already supplies us the information about the nonequilibrium evolution of the anharmonic oscillator when it is coupled to a thermal bath. For the purpose of this paper the dynamics of the real-time two-point functions of the anharmonic oscillator is of special interest.

\section{B. Real-time two-point functions}

We now give a brief derivation via the functional method of the first-order correction to the two-point functions of the anharmonic oscillator. Further details can be found in [28]. For a quartic anharmonic potential,

$$
V(\chi)=\frac{\lambda}{4 !} \chi^{4}, \quad \text { with } \quad \lambda>0,
$$

the first-order correction of the generating functional is given by

$$
\begin{aligned}
\mathcal{Z}_{1}[j]= & -i \lambda \int_{0}^{t} d s\left\{\frac{1}{2 !} \mathfrak{\Im}_{q}(s) G_{H, 0}^{(\chi)}(s, s) \Xi(s) \mathcal{Z}\right. \\
& \left.+\frac{1}{3 !} \mathfrak{\Im}_{q}(s) \Xi^{3}(s) \mathcal{Z}+\frac{1}{4 !} \mathfrak{\Im}_{q}^{3}(s) \Xi(s) \mathcal{Z}\right\},
\end{aligned}
$$

after carrying out the functional derivatives according to (2.7) and (2.8), where $\mathfrak{\Im}_{q}$ and $\Xi$ are given by

$$
\begin{aligned}
& \mathfrak{\Im}_{q}(s)=\int_{0}^{t} d s^{\prime} G_{R, 0}^{(\chi)}\left(s^{\prime}-s\right) j_{q}\left(s^{\prime}\right), \\
& \Xi[j ; \tau)=i \int_{0}^{t} d s^{\prime} G_{H, 0}^{(\chi)}\left(\tau, s^{\prime}\right) j_{q}\left(s^{\prime}\right)+\int_{0}^{t} d s^{\prime} G_{R, 0}^{(\chi)}\left(\tau-s^{\prime}\right) j_{r}\left(s^{\prime}\right) .
\end{aligned}
$$

The real-time Green's functions of the anharmonic oscillator can be constructed from the path-ordered two-point functions,

$$
\left\langle\mathcal{P} \chi(\tau) \chi\left(\tau^{\prime}\right)\right\rangle= \begin{cases}\left\langle\mathcal{T} \chi(\tau) \chi\left(\tau^{\prime}\right)\right\rangle, & \tau \in C_{+} \& \tau^{\prime} \in C_{+}, \\ \left\langle\chi\left(\tau^{\prime}\right) \chi(\tau)\right\rangle, & \tau \in C_{+} \& \tau^{\prime} \in C_{-}, \\ \left\langle\chi(\tau) \chi\left(\tau^{\prime}\right)\right\rangle, & \tau \in C_{-} \& \tau^{\prime} \in C_{+}, \\ \left\langle\mathcal{T}^{*} \chi(\tau) \chi\left(\tau^{\prime}\right)\right\rangle, & \tau \in C_{-} \& \tau^{\prime} \in C_{-},\end{cases}
$$

where $C_{+/-}$represents the forward/backward time branch and $\mathcal{T}, \mathcal{T}^{*}$ denote time-ordering and anti-time-ordering. Thus, the Feynman propagator of the anharmonic oscillator can be evaluated as the second derivatives of the generating functional with respect to $j_{+}$at two different times, that is, with $0<\tau, \tau^{\prime}<t$. Its first-order correction is then

$$
\left\langle\mathcal{T} \chi(\tau) \chi\left(\tau^{\prime}\right)\right\rangle^{(1)}=-\left.\frac{1}{\mathcal{Z}[j ; t)} \frac{\delta^{2} \mathcal{Z}_{1}[j ; t)}{\delta j_{+}(\tau) \delta j_{+}\left(\tau^{\prime}\right)}\right|_{\substack{j=0 \\ q=0}},
$$

which, with the help of (2.9), becomes

$$
\begin{aligned}
\left\langle\mathcal{T} \chi(\tau) \chi\left(\tau^{\prime}\right)\right\rangle^{(1)} & \\
= & \lambda \int_{0}^{t} d s\left\{-\frac{1}{2} G_{R, 0}^{(\chi)}(\tau-s) G_{H, 0}^{(\chi)}(s, s) G_{H, 0}^{(\chi)}\left(s, \tau^{\prime}\right)\right. \\
& -\frac{1}{2} G_{R, 0}^{(\chi)}\left(\tau^{\prime}-s\right) G_{H, 0}^{(\chi)}(s, s) G_{H, 0}^{(\chi)}(s, \tau) \\
& +\frac{i}{4}\left[G_{R, 0}^{(\chi)}(\tau-s) G_{H, 0}^{(\chi)}(s, s) G_{R, 0}^{(\chi)}\left(s-\tau^{\prime}\right)\right. \\
& \left.\left.+G_{R, 0}^{(\chi)}\left(\tau^{\prime}-s\right) G_{H, 0}^{(\chi)}(s, s) G_{R, 0}^{(\chi)}(s-\tau)\right]\right\} .
\end{aligned}
$$

This allows us to read off [28] the first-order corrections of the Hadamard function and the retarded Green's function of the anharmonic oscillator,

$$
\begin{aligned}
G_{H, 1}^{(\chi)}\left(\tau, \tau^{\prime}\right)= & -\frac{\lambda}{2} \int_{0}^{t} d s\left[G_{R, 0}^{(\chi)}(\tau-s) G_{H, 0}^{(\chi)}(s, s) G_{H, 0}^{(\chi)}\left(s, \tau^{\prime}\right)\right. \\
& \left.+G_{R, 0}^{(\chi)}\left(\tau^{\prime}-s\right) G_{H, 0}^{(\chi)}(s, s) G_{H, 0}^{(\chi)}(s, \tau)\right], \quad(2.13) \\
G_{R, 1}^{(\chi)}\left(\tau, \tau^{\prime}\right)= & -\frac{\lambda}{2} \int_{0}^{t} d s G_{R, 0}^{(\chi)}(\tau-s) G_{H, 0}^{(\chi)}(s, s) G_{R, 0}^{(\chi)}\left(s-\tau^{\prime}\right),
\end{aligned}
$$

with $0<\tau^{\prime} \leq \tau<t$. The Green's functions [28] $G_{R, 0}^{(\chi)}\left(\tau-\tau^{\prime}\right)$ and $G_{H, 0}^{(\chi)}\left(\tau, \tau^{\prime}\right)$ are the zeroth-order Green's functions of the anharmonic oscillator, that is, the Green's functions of the harmonic oscillator coupled to the scalar field. Equations (2.13) and (2.14) imply that these first-order corrections in general are not stationary.

However, it can be shown [28] that the first-order corrections of the retarded Green's function and the Hadamard function of the anaharmonic oscillator will become stationary at late times, as their zeroth-order counterparts do. We then have

$$
\begin{aligned}
G_{H, 1}^{(\chi)}\left(\tau, \tau^{\prime}\right) & =G_{H, 1}^{(\chi)}\left(\tau-\tau^{\prime}\right), \\
G_{R, 1}^{(\chi)}\left(\tau, \tau^{\prime}\right) & =G_{R, 1}^{(\chi)}\left(\tau-\tau^{\prime}\right),
\end{aligned}
$$

for $\gamma^{-1} \ll \tau, \tau^{\prime}$, where $\gamma=e^{2} / 8 \pi m$ is the damping constant. This nice property can be partly traced back to the consequence of the interaction between the oscillator and the quantum field. In the context of the perturbative treatment, we observe that the zeroth-order dynamics of the oscillator is equivalent to a damped harmonic oscillator, driven by a stochastic force, or noise, representing the quantum fluctuations of the field, and inherits its statistical properties, such as spectral density, etc. This noise from the environment imparts a stochastic component in the motion of the oscillator which generates radiation whose backreaction introduces a dissipative force which dampens the oscillator's motion. (See, e.g., [27] for a fuller description.) So long as the displacement of the oscillator and the noise 
force are sufficiently small, it is plausible that the anharmonic oscillator can still relax to a equilibrium state. If the displacement (caused in particular by the classical driving force) is not small enough, then the anharmonic potential may excite the oscillator to a higher energy, counteracting the energy loss due to dissipation. Under such circumstances the dynamics of the nonlinear oscillator can become rather complicated, and the perturbation expansion to the first order may cease to be valid.

It is interesting to note that Eq. (2.14) reveals an interesting non-Markovian behavior. We observe that $G_{H, 0}^{(\chi)}(s, s)$ is proportional to the zeroth-order displacement uncertainty of the oscillator. Thus, (2.14) tells us that the disturbance introduced at time $\tau^{\prime}$ is supposed to propagate to the oscillator at time $\tau$, but it receives additional interference from the oscillator's own motional "noise" at time $s$ earlier than time $\tau$. This behavior certainly depends on the evolutionary history of the oscillator. If this structure persists in higher-order corrections, then the process becomes highly non-Markovian because there will be numerous intermediate history-dependent interferences between any two events. Therefore, whether the higherorder anharmonic corrections of the retarded Green's function can reach stationarity is a much more complex story to tell.

The stationary property of the late-time dynamics of the anharmonic oscillator implies that a fluctuation-dissipation relation exists at the first-order correction of the anharmonic oscillator's Green's functions,

$$
\tilde{G}_{H, 1}^{(\chi)}(\kappa)=\operatorname{coth} \frac{\beta \kappa}{2} \operatorname{Im} \tilde{G}_{R, 1}^{(\chi)}(\kappa),
$$

at late time, in additional to the zeroth-order (linear or harmonic oscillator) counterparts,

$$
\tilde{G}_{H, 0}^{(\chi)}(\kappa)=\operatorname{coth} \frac{\beta \kappa}{2} \operatorname{Im} \tilde{G}_{R, 0}^{(\chi)}(\kappa) .
$$

There is also a corresponding relation for the free scalar field at the initial time,

$$
\tilde{G}_{H, \beta}^{(\phi)}(\kappa)=\operatorname{coth} \frac{\beta \kappa}{2} \operatorname{Im} \tilde{G}_{R, 0}^{(\phi)}(\kappa) .
$$

It is interesting to note [27] that they all have the same proportionality factor $\operatorname{coth} \frac{\beta \kappa}{2}$, which depends on the initial temperature of the scalar field, not the "temperature" of the oscillator. This seems to be a generic feature of the nonequilibrium dynamical descriptions of quantum open systems. The oscillator can inherit this temperature only if the coupling between the oscillator and the bath field is vanishingly weak. Otherwise, the effective temperature of the oscillator will depend $[4,32]$ on the configuration. Equations (2.16) and (2.17) seem to light up the hope that we may still obtain a similar form for the higher-order corrections of the Green's function of the anharmonic oscillator, as long as the perturbative expansion remains valid for all times. We will show in the next section that under certain assumptions, we can indeed give a nonperturbative derivation of the FDR at late times for an anharmonic oscillator coupled to a quantum field bath. Moreover, we can provide a derivation of (2.16) from a more physically transparent perspective.

In fact, the derivation of (2.16) has implied two important conditions: 1) The zeroth-order dynamics has a equilibrium state at late times. This state is approached at exponential time and behaves like an attractor, independent of the initial conditions of the oscillator. 2) The nonstationary components of the two-point functions of a weakly nonlinear oscillator vanish exponentially fast at late times. This condition is related to the first condition regarding the exponential relaxation of the dynamics. We will see that these two conditions are essential to providing the nonperturbative arguments for the anharmonic oscillator in next section.

\section{ENERGY FLOW BETWEEN AN ANHARMONIC OSCILLATOR AND ITS QUANTUM FIELD BATH-NONPERTURBATIVE ARGUMENTS}

From the simultaneous set of Heisenberg equations under consideration,

$$
\begin{gathered}
\ddot{\hat{\chi}}(t)+\omega_{0}^{2} \hat{\chi}(t)+\lambda V^{\prime}[\hat{\chi}(t)]=\frac{e}{m} \hat{\phi}(\mathbf{z}, t), \\
\left(\partial_{t}^{2}-\nabla_{x}^{2}\right) \hat{\phi}(\mathbf{x}, t)=e \hat{\chi}(t) \delta^{(3)}(\mathbf{x}-\mathbf{z}),
\end{gathered}
$$

we have the solution of (3.2) given by

$$
\hat{\phi}(\mathbf{x}, t)=\hat{\phi}_{h}(\mathbf{x}, t)+e \int d^{4} x^{\prime} G_{R, 0}^{(\phi)}\left(\mathbf{x}, t ; \mathbf{x}^{\prime}, s\right) \hat{\chi}(s) \delta^{(3)}\left(\mathbf{x}^{\prime}-\mathbf{z}\right) .
$$

Substituting (3.3) into (3.1) gives the reduced Heisenberg equation for the nonlinear oscillator,

$$
\begin{aligned}
\ddot{\hat{\chi}}(t) & +\omega_{0}^{2} \hat{\chi}(t)+\lambda V^{\prime}[\hat{\chi}(t)]-\frac{e^{2}}{m} \int_{0}^{t} d s G_{R, 0}^{(\phi)}(\mathbf{z}, t ; \mathbf{z}, s) \hat{\chi}(s) \\
& =\frac{e}{m} \hat{\phi}_{h}(\mathbf{z}, t) .
\end{aligned}
$$

Here we have placed the self-coupling strength $\lambda$ outside the nonlinear potential $V$. As we have argued before, the nonlinear potential $V(\hat{\chi})$ must possess certain nice features to possibly ensure a stable and unique final state of the oscillator. The term $e \hat{\phi}_{h}(\mathbf{x}, t)$ on the right-hand side of (3.4) represents the noise force from the field bath. The second term on the other hand accounts for the backaction from the radiation of the scalar field $\phi$ emitted from the nonlinear oscillator. These two terms, originating from the interaction 
between the oscillator and the field, will serve as the conduit for the energy exchange between them $[13,27,28]$.

Let $P_{\xi}$ be the power or energy flow delivered by the noise force,

$$
P_{\xi}(\tau)=\frac{e}{2}\left\langle\left\{\hat{\phi}_{h}(\mathbf{z}, \tau), \dot{\hat{\chi}}(\tau)\right\}\right\rangle,
$$

and $P_{\gamma}$ be the power delivered by the backaction of radiation,

$$
\begin{aligned}
P_{\gamma}(\tau) & =\frac{e^{2}}{2} \int_{0}^{\tau} d s G_{R, 0}^{(\phi)}(\mathbf{z}, t ; \mathbf{z}, s)\langle\{\hat{\chi}(s), \dot{\hat{\chi}}(\tau)\}\rangle+\cdots \\
& =\frac{e^{2}}{2} \int_{0}^{t} d s G_{R, 0}^{(\phi)}(\mathbf{z}, t ; \mathbf{z}, s) \frac{d}{d \tau} G_{H}^{(\chi)}(s, \tau)+\cdots
\end{aligned}
$$

where $\cdots$ represents contributions associated with frequency renormalization, then the sum of both powers, the net rate of energy exchange between the anharmonic oscillator and the bath, is given by

$$
P_{\xi}(\tau)+P_{\gamma}(\tau)=\frac{e}{2}\langle\{\hat{\phi}(\mathbf{z}, \tau), \dot{\hat{\chi}}(\tau)\}\rangle+\cdots .
$$

We now use the functional method to show that

$$
\begin{aligned}
\langle\{\hat{\phi}(\mathbf{z}, \tau), \dot{\hat{\chi}}(\tau)\}\rangle= & e \int_{0}^{\tau} d s\left\{\frac{d}{d \tau} G_{R}^{(\chi)}(\tau, s) G_{H, \beta}^{(\phi)}(s, \tau)\right. \\
& \left.+G_{R, 0}^{(\phi)}(\tau-s) \frac{d}{d \tau} G_{H}^{(\chi)}(s, \tau)\right\},
\end{aligned}
$$

such that

$$
\begin{aligned}
& P_{\xi}(\tau)=e^{2} \int_{0}^{t} d s \frac{d}{d \tau} G_{R}^{(\chi)}(\tau, s) G_{H, \beta}^{(\phi)}(s, \tau), \\
& P_{\gamma}(\tau)=\frac{e^{2}}{2} \int_{0}^{\tau} d s G_{R, 0}^{(\phi)}(\mathbf{z}, t ; \mathbf{z}, s) \frac{d}{d \tau} G_{H}^{(\chi)}(s, \tau)+\cdots .
\end{aligned}
$$

This expression holds quite generally without resort to the perturbative expansion. Here we remind that $G^{(\phi)}$ denotes the two-point function of the free field and $G^{(\chi)}$ the twopoint function for the full oscillator dynamics, including backactions from the field.

We can start from (A6) with $\mathcal{Z}$ replaced by the generating functional $\mathcal{Z}_{V}$ of the anharmonic oscillator in (2.7). In this case it is easier to use the functional derivatives with respect to $j_{ \pm}$rather than $j_{q}, j_{r}$. Thus we have, for $0<\tau, \tau^{\prime}<t$,

$$
\begin{aligned}
\left\langle\hat{\phi}(\mathbf{z}, \tau) \hat{\chi}\left(\tau^{\prime}\right)\right\rangle \mathcal{Z}_{V}= & e \int_{0}^{t} d s\left\{\frac{1}{2} G_{R, 0}^{(\phi)}(s-\tau)\left[\frac{\delta^{2}}{i^{2} \delta j_{+}\left(\tau^{\prime}\right) \delta j_{+}(s)}+\frac{\delta^{2}}{i^{2} \delta j_{+}\left(\tau^{\prime}\right) \delta j_{-}(s)}\right] .\right. \\
& +\frac{1}{2} G_{R, 0}^{(\phi)}(\tau-s)\left[\frac{\delta^{2}}{i^{2} \delta j_{+}\left(\tau^{\prime}\right) \delta j_{+}(s)}-\frac{\delta^{2}}{i^{2} \delta j_{+}\left(\tau^{\prime}\right) \delta j_{-}(s)}\right] \\
& \left.+i G_{H, \beta}^{(\phi)}(\tau, s)\left[\frac{\delta^{2}}{i^{2} \delta j_{+}\left(\tau^{\prime}\right) \delta j_{+}(s)}+\frac{\delta^{2}}{i^{2} \delta j_{+}\left(\tau^{\prime}\right) \delta j_{-}(s)}\right]\right\} \mathcal{Z}_{V} \\
= & e \int_{0}^{t} d s\left\{-\frac{i}{2} G_{R, 0}^{(\phi)}(s-\tau)\left[G_{F}^{(\chi)}\left(\tau^{\prime}, s\right)-G_{<}^{(\chi)}\left(\tau^{\prime}, s\right)\right]-\frac{i}{2} G_{R, 0}^{(\phi)}(\tau-s)\left[G_{F}^{(\chi)}\left(\tau^{\prime}, s\right)+G_{<}^{(\chi)}\left(\tau^{\prime}, s\right)\right]\right. \\
& \left.+G_{H, \beta}^{(\phi)}(\tau, s)\left[G_{F}^{(\chi)}\left(\tau^{\prime}, s\right)-G_{<}^{(\chi)}\left(\tau^{\prime}, s\right)\right]\right\} \mathcal{Z}_{V} .
\end{aligned}
$$

Now we will use the identities,

$$
\begin{array}{r}
G_{F}\left(t, t^{\prime}\right)=\frac{1}{2} G_{R}\left(t, t^{\prime}\right)+\frac{1}{2} G_{R}\left(t^{\prime}, t\right)+i G_{H}\left(t, t^{\prime}\right), \\
G_{F}\left(t, t^{\prime}\right)+G_{<}\left(t, t^{\prime}\right)=G_{R}\left(t^{\prime}, t\right)+2 i G_{H}\left(t, t^{\prime}\right),
\end{array}
$$

where, for the case of $\hat{\chi}$, we define the various two-point functions by

$$
\begin{aligned}
& G_{R}^{(\chi)}\left(t, t^{\prime}\right)=i \theta\left(t-t^{\prime}\right)\left\langle\left[\chi(t), \chi\left(t^{\prime}\right)\right]\right\rangle, \\
& G_{H}^{(\chi)}\left(t, t^{\prime}\right)=\frac{1}{2}\left\langle\left\{\chi(t), \chi\left(t^{\prime}\right)\right\}\right\rangle, \\
& G_{F}^{(\chi)}\left(t, t^{\prime}\right)=i \theta\left(t-t^{\prime}\right)\left\langle\chi(t) \chi\left(t^{\prime}\right)\right\rangle+i \theta\left(t^{\prime}-t\right)\left\langle\chi\left(t^{\prime}\right) \chi(t)\right\rangle .
\end{aligned}
$$

Equation (3.11) then becomes

$$
\begin{aligned}
\left\langle\hat{\phi}(\mathbf{z}, \tau) \hat{\chi}\left(\tau^{\prime}\right)\right\rangle= & e \int_{0}^{t} d s\left\{G_{R}^{(\chi)}\left(\tau^{\prime}, s\right) G_{H, \beta}^{(\phi)}(s, \tau)\right. \\
& +G_{R, 0}^{(\phi)}(\tau-s) G_{H}^{(\chi)}\left(s, \tau^{\prime}\right) \\
& -\frac{i}{2} G_{R}^{(\chi)}\left(\tau^{\prime}, s\right) G_{R, 0}^{(\phi)}(s-\tau) \\
& \left.-\frac{i}{2} G_{R, 0}^{(\phi)}(\tau-s) G_{R}^{(\chi)}\left(s, \tau^{\prime}\right)\right\}
\end{aligned}
$$

where $G_{R, 0}^{(\phi)}$ represents the retarded Green's function of the free linear scalar field, while $G_{R}^{(\chi)}$ denotes the full retarded 
Green's function of the nonlinear oscillator, including the influences from the field. We use $G_{R, 0}^{(\chi)}$ for the corresponding retarded Green's function of the linear oscillator, or the zeroth-order contribution of the anharmonic oscillator.

Thus, in the coincident limit $\tau^{\prime} \rightarrow \tau$, we obtain

$$
\begin{aligned}
\langle\hat{\phi}(\mathbf{z}, \tau) \dot{\hat{\chi}}(\tau)\rangle= & e \int_{0}^{t} d s\left\{\frac{d}{d \tau} G_{R}^{(\chi)}(\tau, s) G_{H, \beta}^{(\phi)}(s, \tau)+G_{R, 0}^{(\phi)}(\tau-s) \frac{d}{d \tau} G_{H}^{(\chi)}(s, \tau)\right. \\
& \left.-\frac{i}{2} \frac{d}{d \tau} G_{R}^{(\chi)}(\tau, s) G_{R, 0}^{(\phi)}(s-\tau)-\frac{i}{2} G_{R, 0}^{(\phi)}(\tau-s) \frac{d}{d \tau} G_{R}^{(\chi)}(s, \tau)\right\} \\
= & e \int_{0}^{t} d s\left\{\frac{d}{d \tau} G_{R}^{(\chi)}(\tau, s) G_{H, \beta}^{(\phi)}(s, \tau)+G_{R, 0}^{(\phi)}(\tau-s) \frac{d}{d \tau} G_{H}^{(\chi)}(s, \tau)\right\},
\end{aligned}
$$

because in the last two terms on the right-hand side of the first equal sign, according to the definition of the retarded Green's function, the variable $s$ is both greater and smaller than $\tau$, implying that $\tau=s$ and one of the retarded functions must be zero because $G_{R}(\tau, \tau)=0$ by definition. Therefore we have derived (3.8) and obtained a general expression for the net rate of energy exchange between the anharmonic oscillator and the bath field in terms of the two-point functions of the anharmonic oscillator and those of the free scalar field. It is interesting to know that in general the anharmonic oscillator is not a Gaussian system, so two-point functions or the second moments alone are not sufficient to describe its full statistics, but the higher-point functions do not manifestly appear in the derivation. Equations (3.9) or (3.15) will be used to find the rate of energy exchange between the anharmonic oscillator and the field bath. We will argue that under certain conditions, if the motion of the anharmonic oscillator reaches equilibration, then the vanishing of the net energy exchange will imply a fluctuation-dissipation relation. The converse also holds.

In the special case of the nonequilibrium linear oscillator, its retarded Green's function is stationary, but its Hadamard function in general is not. However, we can show that at late times the nonstationary component of the Hadamard function will be exponentially suppressed such that the Hadamard function becomes stationary. This enables us to show the existence of an FDR for the linear oscillator, which in turn implies that (3.8) vanishes for the linear oscillator coupled to the field bath at any oscillator-field coupling strength. It allows for the power balance and the existence of a stable equilibrium state. However, we can not draw such general conclusions for the anharmonic oscillator. Since in general the driven, damped anharmonic oscillator does not necessarily have a unique stable equilibrium state and quantum chaos can emerge, it is futile to pursue the same line of argument valid for the linear oscillator to seek a general proof of the power balance or existence of the equilibrium state for the anharmonic oscillator. We need stronger constraints on the configurations of the anharmonic oscillator for a equilibrium state to exist. Thus we will use a weaker argument for the vanishing of (3.8).

Before we proceed, we comment on the construction of the power operator by the canonical operator approach for the anharmonic oscillator. For the linear oscillator we use symmetric ordering to write down the operator for the power then compute its expectation value. This procedure may not work for the nonlinear oscillator. Take for example the quartic potential for the anharmonic oscillator. The power associated with the first two terms in (3.4), according to the symmetric ordering, is given by

$$
\frac{1}{2}\left\langle\left\{\ddot{\hat{\chi}}(t)+\omega^{2} \hat{\chi}(t), \dot{\hat{\chi}}(t)\right\}\right\rangle=\frac{d}{d t}\left[\frac{m}{2} \dot{\hat{\chi}}^{2}(t)+\frac{m \omega_{0}^{2}}{2} \hat{\chi}^{2}(t)\right],
$$

but for the power associated with the nonlinear restoring force, we may naïvely conclude that

$$
\frac{1}{2}\left\langle\left\{\hat{\chi}^{3}(t), \dot{\hat{\chi}}(t)\right\}\right\rangle \neq \frac{1}{4} \frac{d}{d t} \hat{\chi}^{4}(t),
$$

because the right-hand side in fact is

$$
\begin{aligned}
\frac{1}{4} \frac{d}{d t} \hat{\chi}^{4}(t)= & \frac{1}{4}\left[\hat{\chi}^{3}(t) \dot{\hat{\chi}}(t)+\hat{\chi}^{2}(t) \dot{\hat{\chi}}(t) \hat{\chi}(t)\right. \\
& \left.+\hat{\chi}(t) \dot{\hat{\chi}}(t) \hat{\chi}^{2}(t)+\dot{\hat{\chi}}(t) \hat{\chi}^{3}(t)\right] \\
= & {\left[\hat{\chi}^{3}(t) \dot{\hat{\chi}}(t)\right]_{\mathrm{w}}, }
\end{aligned}
$$

that is, the Weyl ordering or fully symmetrized ordering. In general, the Weyl ordering of a product of conjugated operators is not equivalent to the symmetric ordering; however, there is an exception, namely, when the product of the conjugated operators takes on the form $\hat{\chi}^{n} \hat{p}$ with $n \in \mathbb{N}$ for the conjugated pair $(\hat{\chi}, \hat{p})$. Thus, in this case, both orderings are compatible, and we can use either ordering to construct the power operator and rewrite the left-hand side of (3.4) as 
$\frac{d}{d t}\left\{\frac{m}{2} \dot{\hat{\chi}}^{2}(t)+\frac{m \omega^{2}}{2} \hat{\chi}^{2}(t)+\lambda V[\hat{\chi}(t)]\right\}=P_{\xi}(\tau)+P_{\gamma}(\tau)$,

where we have introduced the renormalized frequency $\omega$.

Since it is not possible to prove the existence of a stable equilibrium state for the general configurations of the anharmonic oscillator, we take a step down; i.e., we consider only those cases when the dynamics of the anharmonic oscillator can indeed reach a stable equilibrium state at late times. Namely, the energy exchange between the oscillator and the field bath will come into equilibrium. That the rate of the energy exchange will become zero at late times gives

$$
\begin{aligned}
\lim _{\tau \rightarrow \infty} P_{\xi}(\tau)+P_{\gamma}(\tau)= & \lim _{\tau \rightarrow \infty} e^{2} \int_{0}^{\tau} d s\left\{\frac{d}{d \tau} G_{R}^{(\chi)}(\tau, s) G_{H, \beta}^{(\phi)}(s, \tau)\right. \\
& \left.+G_{R, 0}^{(\phi)}(\tau-s) \frac{d}{d \tau} G_{H}^{(\chi)}(s, \tau)\right\}+\cdots \\
= & 0
\end{aligned}
$$

after we have subtracted the contribution corresponding to frequency renormalization. We set forth to show that this energy balance implies a fluctuation-dissipation relation for the anharmonic oscillator.

We first deal with the frequency renormalization more explicitly by introducing a new kernel function $\Gamma_{R, 0}^{(\phi)}(t)$ for the free field,

$$
G_{R, 0}^{(\phi)}(\mathbf{z}, t ; \mathbf{z}, s) \equiv G_{R, 0}^{(\phi)}(t-s)=\frac{d}{d s} \Gamma_{R, 0}^{(\phi)}(t-s) .
$$

After an integration by parts, we find

$$
\begin{aligned}
\int_{0}^{\tau} & d s G_{R, 0}^{(\phi)}(\tau-s) \frac{d}{d \tau} G_{H}^{(\chi)}(s, \tau) \\
= & \int_{0}^{\tau} d s \frac{d}{d s} \Gamma_{R, 0}^{(\phi)}(\tau-s) \frac{d}{d \tau} G_{H}^{(\chi)}(s, \tau) \\
= & \Gamma_{R, 0}^{(\phi)}(0) \frac{d}{d \tau}\left(\frac{\hat{\chi}^{2}(\tau)}{2}\right)-\Gamma_{R, 0}^{(\phi)}(\tau) \frac{d}{d \tau} G_{H}^{(\chi)}(0, \tau) \\
& -\int_{0}^{\tau} d s \Gamma_{R, 0}^{(\phi)}(\tau-s) \frac{d^{2}}{d s d \tau} G_{H}^{(\chi)}(s, \tau) .
\end{aligned}
$$

For a scalar field bath, the kernel $\Gamma_{R, 0}^{(\phi)}(t)$ is proportional to the delta function $\delta(t)$, so the second term on the right-hand side vanishes. Now it is clear to see that the first term corresponds to frequency renormalization, and it will be fused into the bare frequency $\omega_{0}$. Thus, we arrive at

$$
\begin{aligned}
P_{\xi}(\tau)+P_{\gamma}(\tau)= & e^{2} \int_{0}^{\tau} d s\left\{\frac{d}{d \tau} G_{R}^{(\chi)}(\tau, s) G_{H, \beta}^{(\phi)}(s, \tau)\right. \\
& \left.-\Gamma_{R, 0}^{(\phi)}(\tau-s) \frac{d^{2}}{d s d \tau} G_{H}^{(\chi)}(s, \tau)\right\} .
\end{aligned}
$$

The assumed existence of the stable equilibrium state implies that at late times the two-point functions of the oscillator will become stationary. However, this property still cannot allow us to write (3.23) as

$$
\begin{aligned}
P_{\xi}(\tau)+P_{\gamma}(\tau) \stackrel{?}{=} & e^{2} \int_{0}^{\tau} d s\left\{\frac{d}{d \tau} G_{R}^{(\chi)}(\tau-s) G_{H, \beta}^{(\phi)}(\tau-s)\right. \\
& \left.-\Gamma_{R, 0}^{(\phi)}(\tau-s) \frac{d^{2}}{d s d \tau} G_{H}^{(\chi)}(\tau-s)\right\},
\end{aligned}
$$

for sufficiently large $\tau$. Although the nonstationary component of the oscillator's two-point functions decay to zero when both time arguments are large, its contribution to the integral in (3.23) may remain substantial if it does not decay fast enough. In the case of the linear oscillator, the contribution of the nonstationary component diminishes exponentially fast if the nonstationarity originates from the bilinear interaction with the field bath, not from the initial nonstationary state like a squeezed state. We can show that the equal sign in (3.24) holds for a linear oscillator.

For the nonlinear oscillator, we need to put it as an assumption: consider the case that the nonstationary component of the oscillator's two-point function does not contribute to the integral in (3.23), thus validating (3.24). Under this assumption, we can write the integral in (3.24) as

$$
\begin{gathered}
\int_{0}^{\tau} d s\left\{\frac{d}{d \tau} G_{R}^{(\chi)}(\tau-s) G_{H, \beta}^{(\phi)}(\tau-s)+\Gamma_{R, 0}^{(\phi)}(\tau-s) \frac{d^{2}}{d \tau^{2}} G_{H}^{(\chi)}(\tau-s)\right\} \\
=\int_{-\infty}^{\tau} d y\left\{\frac{d}{d y} G_{R}^{(\chi)}(y) G_{H, \beta}^{(\phi)}(y)+\Gamma_{R, 0}^{(\phi)}(y) \frac{d^{2}}{d y^{2}} G_{H}^{(\chi)}(y)\right\}, \\
y=\tau-s,
\end{gathered}
$$

due to the retardation property of the kernel function. In the large $\tau$ limit, we have

$$
\begin{gathered}
\lim _{\tau \rightarrow \infty} \int_{-\infty}^{\tau} d y\left\{\frac{d}{d y} G_{R}^{(\chi)}(y) G_{H, \beta}^{(\phi)}(y)+\Gamma_{R, 0}^{(\phi)}(y) \frac{d^{2}}{d y^{2}} G_{H}^{(\chi)}(y)\right\} \\
=i \int_{-\infty}^{\infty} \frac{d \kappa}{2 \pi} \kappa\left\{\tilde{G}_{R}^{(\chi) *}(\kappa) \tilde{G}_{H, \beta}^{(\phi)}(\kappa)-\tilde{G}_{R, 0}^{(\phi) *}(\kappa) \tilde{G}_{H}^{(\chi)}(\kappa)\right\}
\end{gathered}
$$

since $\tilde{G}_{R, 0}^{(\phi)}(\kappa)=i \kappa \tilde{\Gamma}_{R, 0}^{(\phi)}(\kappa)$. The Fourier transform $\tilde{f}(\kappa)$ of the function $f(\tau)$ is defined by

$$
f(\tau)=\int_{-\infty}^{\infty} \frac{d \kappa}{2 \pi} \tilde{f}(\kappa) e^{-i \kappa \tau}
$$

The kernel functions of the free field satisfy the FDR,

$$
\tilde{G}_{H, \beta}^{(\phi)}(\kappa)=\operatorname{coth} \frac{\beta \kappa}{2} \operatorname{Im} \tilde{G}_{R, 0}^{(\phi)}(\kappa),
$$


if the field is initially in the thermal state. This implies that (3.26) becomes

$=\int_{-\infty}^{\infty} \frac{d \kappa}{2 \pi} \kappa\left\{\operatorname{coth} \frac{\beta \kappa}{2} \operatorname{Im} \tilde{G}_{R}^{(\chi)}(\kappa)-\tilde{G}_{H}^{(\chi)}(\kappa)\right\} \operatorname{Im} \tilde{G}_{R, 0}^{(\phi)}(\kappa)$,

where we have used the properties that $\tilde{G}_{H}(\kappa), \operatorname{Re} \tilde{G}_{R}(\kappa)$ are even with respect to $\kappa$ but $\operatorname{Im} \tilde{G}_{R}(\kappa)$ is an odd function of $\kappa$. Thus the condition that at late times $P_{\xi}+P_{\gamma}$ vanishes implies an FDR for the anharmonic oscillator,

$$
\tilde{G}_{H}^{(\chi)}(\kappa)=\operatorname{coth} \frac{\beta \kappa}{2} \operatorname{Im} \tilde{G}_{R}^{(\chi)}(\kappa) .
$$

This is a nonperturbative result but it requires two rather strong assumptions: 1) a stable equilibrium state exists at late times, and 2) the nonstationary component of the anharmonic oscillator's two-point function has negligible contribution to the integral in (3.23) at late times. These two assumptions can be directly shown to be true for the linear oscillator, but it is not clear yet under what conditions they also hold for the anharmonic oscillator. Nonetheless, in the conceptual framework of quantum open systems the FDR (3.30) derived in the context of nonequilibrium dynamics (as opposed to linear response) for an anharmonic oscillator coupled to a field bath registers a deep connection between equilibration of the system with its environment, balance of energy flow and stationarity in the late-time dynamics. In the next section, we shall use perturbative calculations to assess these two assumptions.

If the assumption that the nonstationary component of the two-point function of the nonlinear oscillator vanishes sufficiently fast is lifted, then we do not have (3.24). Thus the existence of the equilibrium state at late times will instead imply an integral FDR of the anharmonic oscillator like

$$
\begin{aligned}
\lim _{\tau \rightarrow \infty} & \int_{0}^{\tau} d s\left\{\frac{d}{d \tau} G_{R}^{(\chi)}(\tau, s) G_{H, \beta}^{(\phi)}(s, \tau)\right. \\
& \left.-\Gamma_{R, 0}^{(\phi)}(\tau-s) \frac{d^{2}}{d s d \tau} G_{H}^{(\chi)}(s, \tau)\right\}=0,
\end{aligned}
$$

in the time domain, together with (3.28), rather than an algebraic relation (3.30) in the frequency domain.

\section{PERTURBATIVE ASSESSMENT OF ASSUMPTIONS MADE IN THE NONPERTURBATIVE PROOF}

In the previous section, we have formulated a nonperturbative argument to establish the FDR of the anharmonic oscillator, coupled to a thermal field based on a few assumptions. Now we would like to carry out a perturbative calculation for a weakly anharmonic oscillator as an example to examine the conditions which lead to equilibration and the validity of the assumptions invoked. In particular, we will explicitly show how the contributions from the nonstationary components of the oscillator's two-point functions are suppressed by the damping mechanism due to the interaction of the system oscillator with its quantum field environment. This is the second assumption used in the nonperturbative derivation of the FDR, and our result indicates that this assumption seems less essential in the open-system configuration under study.

An important signature for the presence of a stable equilibrium state in the motion of the anharmonic oscillator coupled with a quantum field is that the rate of energy exchange between the oscillator and the field must be balanced such that the net energy flow approaches zero at late times. This condition is not easy to verify for an anharmonic oscillator due to the lack of complete late-time analytical expressions of its observables. In general, results based on the perturbative expansion are not reliable at large evolution time, especially when the nonlinear system is driven by a periodic source. The error accumulation, the secular evolution, and the onset of chaos phenomena associated with the nonlinear system often limit the perturbative calculations to the short time regime. Nonetheless, perturbative treatments may still be applicable to some configurations, restrictive as they may be. One such configuration is the small-amplitude oscillation of a weakly anharmonic oscillator coupled to a low-temperature quantum-field bath, in which the small-amplitude and the weak nonlinearity warrant a perturbative treatment, and the backaction from the field bath induces only a weak stochastic noise and damping. If the anharmonic potential is such that $\chi=0$ remains the unique global minimum of the potential and the other local minima are located far away from $\chi=0$ whereby tunneling is suppressed, the damping dynamics in the system resulting from its interaction with the field would be enough to confine the latetime motion of such a configuration around the global minimum at $\chi=0$.

This may not be the most interesting scenario, but it shows that even perturbative treatment may provide some meaningful description of the late-time dynamics. With the help of the functional method discussed in Appendix, we find the first-order correction to $\langle\hat{\phi}(\mathbf{z}, \tau) \dot{\hat{\chi}}(\tau)\rangle$ given by

$$
\begin{aligned}
\langle\hat{\phi}(\mathbf{z}, \tau) \dot{\hat{\chi}}(\tau)\rangle^{(1)}= & e \int_{0}^{t} d s\left\{G_{R, 0}^{(\phi)}(\tau-s)\left[\frac{d}{d \tau} G_{H, 1}^{(\chi)}(s, \tau)\right]\right. \\
& \left.+\left[\frac{d}{d \tau} G_{R, 1}^{(\chi)}(\tau, s)\right] G_{H, 0}^{(\phi)}(s, \tau)\right\}
\end{aligned}
$$

so that from (3.8) we have 


$$
\begin{aligned}
P_{\xi}^{(1)}(\tau)+P_{\gamma}^{(1)}(\tau)= & e^{2} \int_{0}^{\tau} d s\left\{G_{R, 0}^{(\phi)}(\tau-s)\left[\frac{d}{d \tau} G_{H, 1}^{(\chi)}(s, \tau)\right]\right. \\
& \left.+\left[\frac{d}{d \tau} G_{R, 1}^{(\chi)}(\tau, s)\right] G_{H, 0}^{(\phi)}(s-\tau)\right\}+\cdots
\end{aligned}
$$

with

$$
\begin{aligned}
& P_{\xi}^{(1)}(\tau)=e^{2} \int_{0}^{\tau} d s\left[\frac{d}{d \tau} G_{R, 1}^{(\chi)}(\tau, s)\right] G_{H, 0}^{(\phi)}(s-\tau), \\
& P_{\gamma}^{(1)}(\tau)=e^{2} \int_{0}^{\tau} d s G_{R, 0}^{(\phi)}(\tau-s)\left[\frac{d}{d \tau} G_{H, 1}^{(\chi)}(s, \tau)\right]+\cdots,
\end{aligned}
$$

where $\cdots$ represents contributions related to the frequency renormalization, and the superscript (1) denotes the correction of first order in the self-coupling constant $\lambda$ in the anharmonic potential. Note that we have implicitly assumed that the initial state of the massless scalar field $\phi$ is a stationary state.

Although we have shown [28] that the first-order correction of the kernel functions $G_{R, 1}^{(\chi)}\left(\tau, \tau^{\prime}\right)$ and $G_{H, 1}^{(\chi)}\left(\tau, \tau^{\prime}\right)$ of the $\chi^{4}$ anharmonic oscillator become stationary when $\tau$ and $\tau^{\prime}$ are sufficiently large, we still cannot replace $G_{R, 1}^{(\chi)}(\tau, s)$ and $G_{H, 1}^{(\chi)}(s, \tau)$ in the integrals by $G_{R, 1}^{(\chi)}(\tau-s)$ and $G_{H, 1}^{(\chi)}(s-\tau)$ in the late-time limit $\tau \rightarrow \infty$ because the other time variable $s$ still ranges from 0 to $\tau \rightarrow \infty$. We need to show that the contributions from their nonstationary components to the integrals are negligible.

For $P_{\gamma}^{(1)}$, the arguments is rather straightforward. Since in [28] we have argued that for sufficiently large $\tau$, the nonstationary component of $G_{H, 1}^{(\chi)}(s, \tau)$ will contain a factor like $e^{-\gamma s}$, and since we observe that for a massless scalar field $\phi$, its retarded function $G_{R, 0}^{(\phi)}(\tau-s)$ drops to zero rapidly when $s$ deviates from $\tau$, we conclude that the dominant contribution of the integral (4.4) will come from the values of $s$ in the vicinity of $\tau$. Thus in the limit $\tau \rightarrow \infty$, we can write $G_{H, 1}^{(\chi)}(s, \tau)$ in (4.4) as approaching $G_{H, 1}^{(\chi)}(s-\tau)$.

As for $P_{\xi}^{(1)}$, let us examine the contribution from the nonstationary component of $G_{R, 1}^{(\chi)}\left(\tau, \tau^{\prime}\right)$. We first observe that by construction in (2.14), $G_{R, 1}^{(\chi)}(\tau, \tau$, ) is given by

$$
\begin{aligned}
G_{R, 1}^{(\chi)}\left(\tau, \tau^{\prime}\right)= & -\frac{\lambda}{2 !} \int_{0}^{\tau} d s G_{R, 0}^{(\chi)}(\tau-s) G_{H, 0}^{(\chi)}(s, s) G_{R, 0}^{(\chi)}\left(s-\tau^{\prime}\right) \\
= & -\frac{\lambda}{4 \Omega^{2}} \int_{0}^{\tau} d s e^{-\gamma\left(\tau-\tau^{\prime}\right)}\left[\cos \Omega\left(\tau+\tau^{\prime}-2 s\right)\right. \\
& \left.-\cos \Omega\left(\tau-\tau^{\prime}\right)\right] G_{H, 0}^{(\chi)}(s, s),
\end{aligned}
$$

where we have substituted the expressions of $G_{R, 0}^{(\chi)}(\tau-s)$. It is useful to write (4.5) as the sum of two integrals $I_{1}$ and $I_{2}$,

$$
\begin{aligned}
& I_{1}=\frac{\lambda}{4 \Omega^{2}} e^{-\gamma\left(\tau-\tau^{\prime}\right)} \cos \Omega\left(\tau-\tau^{\prime}\right) \int_{\tau^{\prime}}^{\tau} d s G_{H, 0}^{(\chi)}(s, s), \\
& I_{2}=-\frac{\lambda}{4 \Omega^{2}} e^{-\gamma\left(\tau-\tau^{\prime}\right)} \int_{\tau^{\prime}}^{\tau} d s \cos \Omega\left(\tau+\tau^{\prime}-2 s\right) G_{H, 0}^{(\chi)}(s, s) .
\end{aligned}
$$

We note [28] that in general $G_{H, 0}^{(\chi)}(s, s)$ take the form,

$$
G_{H, 0}^{(\chi)}(s, s)=\text { const. }+e^{-\gamma s}(\cdots)+e^{-2 \gamma s}(\cdots),
$$

where terms in $(\cdots)$ are sinusoidal in $s$, i.e., $e^{ \pm i \varpi s}$ with some constant $\varpi \in \mathbb{R}$, and "const" represents terms independent of $s$. In addition, generically we have

$$
\begin{aligned}
& \int_{\tau^{\prime}}^{\tau} d s=\tau-\tau^{\prime}, \int_{\tau^{\prime}}^{\tau} d s e^{-\gamma s} e^{i \omega s}=-\frac{e^{-\gamma \tau+i \omega \tau}}{\gamma-i \omega}+\frac{e^{-\gamma \tau^{\prime}+i \omega \tau^{\prime}}}{\gamma-i \omega}, \\
& \int_{\tau^{\prime}}^{\tau} d s \cos \Omega\left(\tau+\tau^{\prime}-2 s\right)=\frac{1}{\Omega} \sin \Omega\left(\tau-\tau^{\prime}\right), \\
& \int_{\tau^{\prime}}^{\tau} d s e^{-\gamma s} e^{i \omega s} \cos \Omega\left(\tau+\tau^{\prime}-2 s\right) \\
& =A e^{\gamma \tau+i \omega \tau} g\left(\tau-\tau^{\prime}\right)+B e^{\gamma \tau^{\prime}+i \omega \tau^{\prime}} g\left(\tau-\tau^{\prime}\right),
\end{aligned}
$$

where $A, B$ are $\tau, \tau^{\prime}$-independent constants, and $g(s)$ is some bounded sinusoidal function of $s$. These points show that the nonstationary terms in $G_{R, 1}^{(\chi)}$ are always exponentially smaller than the stationary terms. Thus the contribution from the nonstationary terms of $G_{R, 1}^{(\chi)}$ to the integral in the definition $P_{\xi}^{(1)}$ for $\tau \rightarrow \infty$ will be typically likewise smaller than the contribution from the stationary terms of $G_{R, 1}^{(\chi)}$. This implies that we can drop the nonstationary terms in $G_{R, 1}^{(\chi)}\left(\tau, \tau^{\prime}\right)$ with negligible errors. In other words, we now can write $G_{R, 1}^{(\chi)}\left(\tau, \tau^{\prime}\right)=G_{R, 1}^{(\chi)}\left(\tau-\tau^{\prime}\right)$ in (4.3) such that

$$
P_{\xi}^{(1)}(\tau)=e^{2} \int_{0}^{\tau} d s\left[\frac{d}{d \tau} G_{R, 1}^{(\chi)}(\tau-s)\right] G_{H, 0}^{(\phi)}(s-\tau),
$$

for large $\tau$, and in this limit the net power becomes

$$
\begin{aligned}
P_{\xi}^{(1)}(\tau)+P_{\gamma}^{(1)}(\tau)= & e^{2} \int_{0}^{\tau} d s\left\{G_{R, 0}^{(\phi)}(\tau-s)\left[\frac{d}{d \tau} G_{H, 1}^{(\chi)}(s-\tau)\right]\right. \\
& \left.+\left[\frac{d}{d \tau} G_{R, 1}^{(\chi)}(\tau-s)\right] G_{H, 0}^{(\phi)}(s-\tau)\right\}+\cdots
\end{aligned}
$$

From the previous arguments, we find that up to the firstorder perturbation of anharmonicity, the second assumption used for the nonperturbative derivation of the FDR holds quite naturally. The exponential suppression of the 
contribution from the nonstationary component is related to the exponential relaxation of the zeroth-order dynamics, which in turn is a consequence of the finite damping force in the equation of motion of the reduced anharmonic system.

Next, following the arguments between (3.21) and (3.30) adapted for the first-order nonlinear correction, and the perturbative FDR (2.16) for the nonlinear oscillator, we conclude that the first-order anharmonic correction of the net power will vanish at late times $\tau \rightarrow \infty$. Thus, it implies that for the configuration we are interested in, the motion of the anharmonic oscillator will eventually be relaxed to a equilibrium state in the weak anhamonicity limit. Moreover, within the validity of this perturbative treatment, both assumptions used for the nonperturbative argument are not needed $a$ priori. The backactions from the bath field naturally enforce these assumptions. Two more observations worth some comments here. In [28], it has been shown that the first-order anharmonic correction of the energy flows at late times are independent of the bath temperature in the high-temperature limit. The hightemperature thermal fluctuations of the bath field, although, linearly enhance with the bath temperature the zeroth-order energy flows between the oscillator and the bath, they do not affect the first-order contribution. Thus it does not seem necessary to restrict the bath to a low-temperature thermal field. Secondly, since the zeroth order dynamics always asymptotically relax to a stable equilibrium state independent of the initial state, we may not need to confine the initial displacement of the oscillator to be small, as long as the (harmonic + anharmonic) potential has one minimum and the perturbation theory remains valid. In the weak anharmonicity limit, the finite damping seems to well herd the oscillator's motion to the equilibrium state. This also bring up the interesting role the environment plays in the dynamics of the nonlinear system. The reasoning used here then adds to the plausibility of the assumption used in the nonperturbative arguments on a broader ground.

\section{SUMMARY}

In this paper, we use the functional method to derive nonperturbative expressions for the energy flows between an anharmonic quantum oscillator and the quantum field bath under nonequilibrium conditions. There is no guarantee that the dynamics of a general nonlinear quantum oscillator will settle down to an equilibrium state. Thus we look at specific cases. In our derivation of the nonperturbative FDR for nonlinear open systems we need to make two assumptions, namely, (1) the nonlinear oscillator evolves to a unique stable equilibrium state at late times, and (2) the nonstationary components of the two-point functions of the nonlinear oscillator has negligible contributions to the energy flows. Under these two assumptions, we find that if the energy flows come to a balance, then a nonperturbative FDR can be derived for the nonlinear oscillator. Since the balance of energy flows has been linked to dynamical equilibration for the case of the linear oscillator, the nonperturbative FDR we derive here points to such a connection. To show this connection concretely, we treat a weakly anharmonic oscillator in the same configuration by the functional perturbative method and show that the second assumption holds quite naturally. Together with the result found in [28], the first assumption can also be lifted for weak anharmonicity. Both can be attributed to finite damping due to the interaction with the bath field.

Our quantum model calculation provides a microscopic perspective to the relation between the dynamical equilibration of a nonlinear system and the balance of energy exchange with its environment. The existence of a nonperturbative FDR for nonlinear quantum systems seems to reinforce the notion that FDRs are indeed categorical relations reflecting the delicate balance between fluctuations in the environment and the induced dissipation in the open system, quite robust in the nature of the system and the details of the environment.

\section{ACKNOWLEDGMENTS}

This work makes use of results obtained when both authors visited the Center for Particle Physics and Field Theory of Fudan University. It is further developed when J. T. H. visited the Maryland Center for Fundamental Physics at the University of Maryland and when B. L. H. visited the National Center for Theoretical Sciences in Hsinchu, Taiwan.

\section{APPENDIX: DERIVATION OF ENERGY FLOW BETWEEN THE OSCILLATOR AND THE BATH FIELD}

To illustrate the functional method we calculate the power $P_{\xi}$ delivered by a massless quantum scalar field $\hat{\phi}(\mathbf{x}, t)$ to the linear (harmonic) oscillator $\hat{\chi}(t)$, which can be viewed as the internal degree of freedom of an UnruhDeWitt detector.

The power delivered by the free field at time $\tau$ is defined by

$$
P_{\xi}(\tau)=\frac{e}{2}\left\langle\left\{\hat{\phi}_{h}(\mathbf{z}, \tau), \hat{\chi}(\tau)\right\}\right\rangle,
$$

where the external degree of freedom $\mathbf{z}$ of the detector gives the fixed location of the detector, and $\hat{\phi}_{h}(\mathbf{z}, t)$ is the free field component, that is, the homogeneous solution of the field equation. Although (A1) is the real part of the coincident limit,

$$
e \lim _{\tau^{\prime} \rightarrow \tau} \frac{d}{d \tau^{\prime}}\left\langle\hat{\phi}_{h}(\mathbf{z}, \tau) \hat{\chi}\left(\tau^{\prime}\right)\right\rangle,
$$

we will use the functional method to derive the expressions for 


$$
\left\langle\hat{\phi}(\mathbf{z}, \tau) \hat{\chi}\left(\tau^{\prime}\right)\right\rangle,
$$

in terms of two-point functions of the oscillator and the free field. Note that (A3) involves the full interacting field $\hat{\phi}(\mathbf{z}, t)$, instead of the free field $\hat{\phi}_{h}(\mathbf{z}, t)$, so the result will contain an additional contribution from the radiation field emitted by the evolving oscillator, that is, $P_{\gamma}(\tau)$. For a linear oscillator it is pretty easy to distinguish the contribution of the free field from that of the radiation field. ${ }^{2}$

Since the expectation value (A3) contains the field operator, it is not obvious a priori how to apply the functional method to the generating functional we have in Sec. II to compute the expectation value. We return to the starting point, noting that (A3) means

$$
\left\langle\hat{\phi}(\mathbf{z}, \tau) \hat{\chi}\left(\tau^{\prime}\right)\right\rangle=\operatorname{Tr}_{\chi \phi}\left\{\hat{\rho}_{\chi \phi}(t) \hat{\phi}(\mathbf{z}, \tau) \hat{\chi}\left(\tau^{\prime}\right)\right\}
$$

where $\hat{\rho}_{\chi \phi}$ is the density operator of the whole system, $0<\tau, \tau^{\prime}<t$ and $t$ can be taken to $+\infty$ for convenience. Introducing a path integral representation, we have

$$
\begin{aligned}
& \left\langle\hat{\phi}(\mathbf{z}, \tau) \hat{\chi}\left(\tau^{\prime}\right)\right\rangle_{j} \mathcal{Z}_{V}[j ; t)=\int_{-\infty}^{\infty} d \chi_{b} d \chi_{b}^{\prime} \delta\left(\chi_{b}-\chi_{b}^{\prime}\right) \int_{-\infty}^{\infty} d \chi_{a} d \chi_{a}^{\prime} \rho_{\chi}\left(\chi_{a}, \chi_{a}^{\prime}, 0\right) \\
& \times \int_{\chi_{a}}^{\chi_{b}} \mathcal{D} \chi_{+} \int_{\chi_{a}^{\prime}}^{\chi_{b}^{\prime}} \mathcal{D} \chi_{-} \chi_{+}\left(\tau^{\prime}\right) \exp \left(i S_{\chi}\left[\chi_{+}, j_{+}\right]-i S_{\chi}\left[\chi_{-}, j_{-}\right]\right) \\
& \times \int_{-\infty}^{\infty} d \phi_{b} d \phi_{b}^{\prime} \delta\left(\phi_{b}-\phi_{b}^{\prime}\right) \int_{-\infty}^{\infty} d \phi_{a} d \phi_{a}^{\prime} \rho_{\phi}\left(\phi_{a}, \phi_{a}^{\prime}, 0\right) \\
& \times \int_{\phi_{a}}^{\phi_{b}} \mathcal{D} \phi_{+} \int_{\phi_{a}^{\prime}}^{\phi_{b}^{\prime}} \mathcal{D} \phi_{-} \phi_{+}(\mathbf{z}, \tau) \exp \left(i S_{I}\left[\chi_{+}, \phi_{+}\right]-i S_{I}\left[\chi_{-}, \phi_{-}\right]+i S_{\phi}\left[\phi_{+}\right]-i S_{\phi}\left[\phi_{-}\right]\right) \\
& =\frac{\delta}{i \delta j_{+}\left(\tau^{\prime}\right)} \int_{-\infty}^{\infty} d \chi_{b} d \chi_{b}^{\prime} \delta\left(\chi_{b}-\chi_{b}^{\prime}\right) \int_{-\infty}^{\infty} d \chi_{a} d \chi_{a}^{\prime} \rho_{\chi}\left(\chi_{a}, \chi_{a}^{\prime}, 0\right) \\
& \times \int_{\chi_{a}}^{\chi_{b}} \mathcal{D} \chi_{+} \int_{\chi_{a}^{\prime}}^{\chi_{b}^{\prime}} \mathcal{D} \chi_{-} \exp \left(i S_{\chi}\left[\chi_{+}, j_{+}\right]-i S_{\chi}\left[\chi_{-}, j_{-}\right]\right) \\
& \times \frac{1}{e} \frac{\delta}{i \delta \chi_{+}(\tau)} \int_{-\infty}^{\infty} d \phi_{b} d \phi_{b}^{\prime} \delta\left(\phi_{b}-\phi_{b}^{\prime}\right) \int_{-\infty}^{\infty} d \phi_{a} d \phi_{a}^{\prime} \rho_{\phi}\left(\phi_{a}, \phi_{a}^{\prime}, 0\right) \\
& \times \int_{\phi_{a}}^{\phi_{b}} \mathcal{D} \phi_{+} \int_{\phi_{a}^{\prime}}^{\phi_{b}^{\prime}} \mathcal{D} \phi_{-} \exp \left(i S_{I}\left[\chi_{+}, \phi_{+}\right]-i S_{I}\left[\chi_{-}, \phi_{-}\right]+i S_{\phi}\left[\phi_{+}\right]-i S_{\phi}\left[\phi_{-}\right]\right) \\
& =\frac{\delta}{i \delta j_{+}\left(\tau^{\prime}\right)} \int_{-\infty}^{\infty} d \chi_{b} d \chi_{b}^{\prime} \delta\left(\chi_{b}-\chi_{b}^{\prime}\right) \int_{-\infty}^{\infty} d \chi_{a} d \chi_{a}^{\prime} \rho_{\chi}\left(\chi_{a}, \chi_{a}^{\prime}, 0\right) \\
& \times \int_{\chi_{a}}^{\chi_{b}} \mathcal{D} \chi_{+} \int_{\chi_{a}^{\prime}}^{\chi_{b}^{\prime}} \mathcal{D} \chi_{-} \exp \left(i S_{\chi}\left[\chi_{+}, j_{+}\right]-i S_{\chi}\left[\chi_{-}, j_{-}\right]\right) \\
& \times\left\{e \int_{0}^{t} d s\left[\frac{1}{2} G_{R, 0}^{(\phi)}(s-\tau) q(s)+G_{R, 0}^{(\phi)}(\tau-s) r(s)+i G_{H, \beta}^{(\phi)}(\tau, s) q(s)\right]\right\} \\
& \times \exp \left\{i e^{2} \int_{0}^{t} d s d s^{\prime}\left[q(s) G_{R, 0}^{(\phi)}\left(s, s^{\prime}\right) r\left(s^{\prime}\right)+\frac{i}{2} q(s) G_{H, \beta}^{(\phi)}\left(s, s^{\prime}\right) q\left(s^{\prime}\right)\right]\right\} \\
& =-e \int_{0}^{t} d s\left\{\left[\frac{1}{4} G_{R, 0}^{(\phi)}(s-\tau) \frac{\delta^{2}}{\delta j_{r}\left(\tau^{\prime}\right) \delta j_{r}(s)}+\frac{i}{2} G_{H, \beta}^{(\phi)}(\tau, s) \frac{\delta^{2}}{\delta j_{r}\left(\tau^{\prime}\right) \delta j_{r}(s)}\right]\right. \\
& +\left[\frac{1}{2} G_{R, 0}^{(\phi)}(\tau-s) \frac{\delta^{2}}{\delta j_{r}\left(\tau^{\prime}\right) \delta j_{q}(s)}+\frac{1}{2} G_{R, 0}^{(\phi)}(s-\tau) \frac{\delta^{2}}{\delta j_{q}\left(\tau^{\prime}\right) \delta j_{r}(s)}\right. \\
& \left.\left.+i G_{H, \beta}^{(\phi)}(\tau, s) \frac{\delta^{2}}{\delta j_{q}\left(\tau^{\prime}\right) \delta j_{r}(s)}\right]+G_{R, 0}^{(\phi)}(\tau-s) \frac{\delta^{2}}{\delta j_{q}\left(\tau^{\prime}\right) \delta j_{q}(s)}\right\} \times \mathcal{Z}_{V}\left[j_{r}, j_{q} ; t\right),
\end{aligned}
$$

\footnotetext{
${ }^{2}$ From Eq. (3.3), we observe that the first term on its right-hand side, the homogeneous solution of the field equation (3.2), describes the free quantum field, while the second term, the inhomogeneous solution, is a radiation field, i.e., Liénard Wiechert potential [33] emitted by the oscillator at $\mathbf{z}$. More discussions can be found in [27].
} 
where the oscillator action $S_{\chi}[\chi, j]$ contains the contributions from the external sources,

$S_{\chi}[\chi, j]=\int_{0}^{t} d s\left\{\frac{m}{2}\left[\dot{\chi}^{2}(s)-\omega_{0}^{2} \chi^{2}(s)\right]-V[\chi(s)]+j(s) \chi(s)\right\}$,

and $V[\chi]$ accounts for the nonlinear potential. Here, be reminded that $G_{R, 0}^{(\phi)}$ and $G_{H, \beta}^{(\phi)}$ individually denotes the retarded Green's function and the Hadamard function of the free field, while $G_{R}^{(\chi)}$ and $G_{H}^{(\chi)}$ are respectively the retarded Green's function and the Hadamard function of the anharmonic oscillator interacting with the scalar field.

Here Eq. (A6) indicates that the insertion of $\phi(\mathbf{z}, \tau)$ is equivalent to taking an additional functional derivative of the combination,

$$
\begin{aligned}
& e \int_{0}^{t} d s\left[\frac{1}{2} G_{R, 0}^{(\phi)}(s-\tau) \frac{\delta}{i \delta j_{r}(s)}+G_{R, 0}^{(\phi)}(\tau-s) \frac{\delta}{i \delta j_{q}(s)}\right. \\
& \left.\quad+i G_{H, \beta}^{(\phi)}(\tau, s) \frac{\delta}{i \delta j_{r}(s)}\right]
\end{aligned}
$$

of the generating functional $\mathcal{Z}_{V}[j ; t)$. Since it originates from the influence action, we can infer that the terms involving the retarded Green's function of the free field $G_{R, 0}^{(\phi)}$ are related to the radiation field, caused by the nontrivial motion of the internal degree of freedom of the detector, while that associated with the Hadamard function of the free field $G_{H, \beta}^{(\phi)}$ pertains to the free field fluctuations. The latter will be what we search for in calculating (A3) and the power delivered by the free field $\hat{\phi}_{h}(\mathbf{z}, t)$.

Let us for the moment consider the simpler case that the nonlinear potential $V[\chi]$ is absent. To evaluate (A6), we need the following identities from previous calculations:

$$
\frac{\delta^{2} \mathcal{Z}[j ; t)}{\delta j_{q}(\tau) \delta j_{q}\left(\tau^{\prime}\right)}=i \frac{\delta \Xi\left[j ; \tau^{\prime}\right)}{\delta j_{q}(\tau)} \mathcal{Z}[j ; t)-\Xi[j ; \tau) \Xi\left[j ; \tau^{\prime}\right) \mathcal{Z}[j ; t),
$$

$$
\frac{\delta^{2} \mathcal{Z}[j ; t)}{\delta j_{q}\left(\tau^{\prime}\right) \delta j_{r}(\tau)}=i \frac{\delta \mathfrak{\Im}_{q}(\tau)}{\delta j_{q}\left(\tau^{\prime}\right)} \mathcal{Z}[j ; t)-\mathfrak{J}_{q}(\tau) \Xi\left[j ; \tau^{\prime}\right) \mathcal{Z}[j ; t),
$$

$$
\frac{\delta^{2} \mathcal{Z}[j ; t)}{\delta j_{q}(\tau) \delta j_{r}\left(\tau^{\prime}\right)}=i \frac{\delta \mathfrak{\Im}_{q}\left(\tau^{\prime}\right)}{\delta j_{q}(\tau)} \mathcal{Z}[j ; t)-\mathfrak{\Im}_{q}\left(\tau^{\prime}\right) \Xi[j ; \tau) \mathcal{Z}[j ; t),
$$

$$
\frac{\delta^{2} \mathcal{Z}[j ; t)}{\delta j_{r}(\tau) \delta j_{r}\left(\tau^{\prime}\right)}=-\mathfrak{\Im}_{q}(\tau) \mathfrak{\Im}_{q}\left(\tau^{\prime}\right) \mathcal{Z}[j ; t)
$$

and $-i \frac{\delta \Xi\left[j ; \tau^{\prime}\right)}{\delta j_{q}(\tau)}=G_{H, 0}^{(\chi)}\left(\tau, \tau^{\prime}\right), \quad \frac{\delta \Im_{q}\left(\tau^{\prime}\right)}{\delta j_{q}(\tau)}=G_{R, 0}^{(\chi)}\left(\tau-\tau^{\prime}\right)$,

with $\mathfrak{\Im}_{q}(s), \Xi[j ; \tau)$ defined by

$$
\begin{aligned}
\mathfrak{\Im}_{q}(s)= & \int_{0}^{t} d s^{\prime \prime} G_{R, 0}^{(\chi)}\left(s^{\prime \prime}-s\right) j_{q}\left(s^{\prime \prime}\right), \\
\Xi[j ; \tau)= & i \int_{0}^{t} d s^{\prime} G_{H, 0}^{(\chi)}\left(\tau, s^{\prime}\right) j_{q}\left(s^{\prime}\right) \\
& +\int_{0}^{t} d s^{\prime} G_{R, 0}^{(\chi)}\left(\tau-s^{\prime}\right) j_{r}\left(s^{\prime}\right) .
\end{aligned}
$$

Thus in the limits $j_{r}, j_{q} \rightarrow 0$, we find that

$$
\begin{aligned}
\left\langle\hat{\phi}(\mathbf{z}, \tau) \hat{\chi}\left(\tau^{\prime}\right)\right\rangle= & e \int_{0}^{\tau^{\prime}} d s G_{R, 0}^{(\chi)}\left(\tau^{\prime}-s\right) G_{H, \beta}^{(\phi)}(s, \tau) \\
& +e \int_{0}^{\tau} d s G_{R, 0}^{(\phi)}(\tau-s) G_{H, 0}^{(\chi)}\left(s, \tau^{\prime}\right) \\
& -i \frac{e}{2} \int_{0}^{\tau} d s G_{R, 0}^{(\phi)}(\tau-s) G_{R, 0}^{(\chi)}\left(s-\tau^{\prime}\right) \\
& -i \frac{e}{2} \int_{0}^{\tau^{\prime}} d s G_{R, 0}^{(\chi)}\left(\tau^{\prime}-s\right) G_{R, 0}^{(\phi)}(s-\tau) .
\end{aligned}
$$

Note that we pick $\phi_{+}$and $\chi_{+}$in (A5), so Eq. (A13) is a form of time-ordered two-point function, instead of the Schwinger type of two-point function. Thus its real part gives the corresponding expectation value of (one half of) the anticommutator, or the Hadamard-like function,

$$
\begin{aligned}
\frac{1}{2}\left\langle\left\{\hat{\phi}(\mathbf{z}, \tau), \hat{\chi}\left(\tau^{\prime}\right)\right\}\right\rangle= & e \int_{0}^{\tau^{\prime}} d s G_{R, 0}^{(\chi)}\left(\tau^{\prime}-s\right) G_{H, \beta}^{(\phi)}(s, \tau) \\
& +e \int_{0}^{\tau} d s G_{R, 0}^{(\phi)}(\tau-s) G_{H, 0}^{(\chi)}\left(s, \tau^{\prime}\right),
\end{aligned}
$$

in which we use the superscripts to distinguish the Green's functions of different subsystems. According to the previous discussions, we in fact need the component $\left\langle\left\{\hat{\phi}_{h}(\mathbf{z}, \tau), \hat{\chi}\left(\tau^{\prime}\right)\right\}\right\rangle$, which is then given by

$\frac{1}{2}\left\langle\left\{\hat{\phi}_{h}(\mathbf{z}, \tau), \hat{\chi}\left(\tau^{\prime}\right)\right\}\right\rangle=e \int_{0}^{\tau^{\prime}} d s G_{R}^{(\chi)}\left(\tau^{\prime}-s\right) G_{H, \beta}^{(\phi)}(s, \tau)$.

Taking the coincident limit of its $\tau^{\prime}$ derivative gives the power delivered by the free field,

$$
P_{\xi}(\tau)=e^{2} \int_{0}^{\tau} d s \dot{G}_{R}^{(\chi)}(\tau-s) G_{H, \beta}^{(\phi)}(s, \tau) .
$$

The $P_{\xi}$ associated with the interaction between the nonlinear oscillator and the bath field, discussed in Sec. III can then be obtained in a similar manner by putting back the nonlinear potential $V$ and replacing the in-in generating functional $\mathcal{Z}$ with $\mathcal{Z}_{V}$ in the earlier derivations. 
[1] R. Kubo, The fluctuation-dissipation theorem, Rep. Prog. Phys. 29, 255 (1966).

[2] R. Kubo, M. Toda, and N. Hashitsume, Statistical Physics II: Nonequilibrium Statistical Mechanics (Springer Verlag, Berlin Heidelberg, 1991).

[3] D. W. Sciama, Thermal and quantum Fluctuations in special and general relativity: An Einstein synthesis, in Centenario di Einstein (Editrici Giunti Barbera Universitaria, Florence, Italy, 1979).

[4] J.-T. Hsiang, C. H. Chou, Y. Subaşı, and B. L. Hu, Quantum thermodynamics from the nonequilibrium dynamics of open systems: Energy, heat capacity, and the third law, Phys. Rev. E 97, 012135 (2018).

[5] J.-T. Hsiang and B. L. Hu, Nonequilibrium steady state in open quantum systems: influence action, stochastic equation and power balance, Ann. Phys. (Amsterdam) 362, 139 (2015).

[6] L. Kadanoff and G. Baym, Quantum Statistical Mechanics (Benjamin, New York, 1962).

[7] S. W. Lovesey, Condensed Matter Physics: Dynamic Correlations (Addison-Wesley, Reading, 1986).

[8] A. L. Fetter and J. D. Walecka, Quantum Theory of Manyparticle Systems (Courier Corporation, Dover, 2003).

[9] P. Candelas and D. W. Sciama, Irreversible Thermodynamics of Black Holes, Phys. Rev. Lett. 38, 1372 (1977); Erratum, Phys. Rev. Lett. 39, 1640 (1977).

[10] E. Mottola, Quantum fluctuation-dissipation theorem for general relativity, Phys. Rev. D 33, 2136 (1986).

[11] B. L. Hu and S. Sinha, Fluctuation-dissipation relation for semiclassical cosmology, Phys. Rev. D 51, 1587 (1995).

[12] G. W. Ford, The fluctuation-dissipation theorem, Contemp. Phys. 58, 244 (2017).

[13] J.-T. Hsiang, B. L. Hu, and S.-Y. Lin, Fluctuation-dissipation and correlation-propagation relations from the nonequilibrium dynamics of detector-quantum field systems, Phys. Rev. D 100, 025019 (2019); J.-T. Hsiang, B. L. Hu, S.-Y. Lin, and K. Yamamoto, Fluctuation-dissipation and correlationpropagation relations in $(1+3) \mathrm{D}$ moving detector-quantum field systems, Phys. Lett. B 795, 694 (2019).

[14] G. F. Eremov, A fluctuation dissipation theorem for nonlinear medium, Zh. Eksp. Teor. Fiz. 55, 2322 (1968), http:// jetp.ac.ru/cgi-bin/dn/e_028_06_1232.pdf.

[15] K. I. Golden, G. Kalman, and M. B. Silevitch, Nonlinear fluctuation-dissipation theorem, J. Stat. Phys. 6, 87 (1972).

[16] R. Zwanzig, Nonlinear generalized Langevin equations, J. Stat. Phys. 9, 215 (1973).

[17] D. C. Langreth, Linear and nonlinear response theory with applications, in Linear and Nonlinear Electron Transport in Solids, edited by J. T. Devreese and V. E. van Doren, NATO Advanced Study Institutes Series (Series B: Physics) Vol. 17 (Springer Verlag, Boston, 1976).

[18] G.-Z. Zhou, Z.-B. Su, B.-L. Hao, and L. Yu, Closed time path Green's functions and critical dynamics, Phys. Rev. B 22, 3385 (1980).

[19] G. N. Bochkov and Y. E. Kuzovlev, Nonlinear fluctuationdissipation relations and stochastic models in nonequilibrium thermodynamics: I. Generalized fluctuation-dissipation theorem, Physica A (Amsterdam) 106, 443 (1981); II. Kinetic potential and variational principles for nonlinear irreversible processes, Physica A (Amsterdam) 106, 480 (1981).

[20] R. L. Stratonovich, Nonlinear Nonequilibrium Thermodynamics I. Linear and Nonlinear Fluctuation-Dissipation Theorems (Springer-Verlag, Berlin Heidelberg, 1992).

[21] E. Wang and U. Heinz, Generalized fluctuation-dissipation theorem for nonlinear response functions, Phys. Rev. D 66 , 025008 (2002).

[22] M. E. Carrington, D. Hou, and R. Kobes, Shear viscosity in $\varphi^{4}$ theory from an extended ladder resummation, Phys. Rev. D 62, 025010 (2000); M. E. Carrington, D. Hou, and J. C. Sowiak, Kubo-Martin-Schwinger conditions for 4-point Green functions at finite temperature, Phys. Rev. D 62, 065003 (2000).

[23] K. Miyazaki and D. R. Reichman, Mode-coupling theory and the fluctuation-dissipation theorem for nonlinear Langevin equations with multiplicative noise, J. Phys. A 38, L343 (2005).

[24] J. Maciejko, J. Wang, and H. Guo, Time-dependent quantum transport far from equilibrium: An exact nonlinear response theory, Phys. Rev. B 74, 085324 (2006).

[25] T. Motz, M. Wiedmann, J. T. Stockburger, and J. Ankerhold, Rectification of heat currents across nonlinear quantum chains: A versatile approach beyond weak thermal contact, New J. Phys. 20, 113020 (2018).

[26] N. Tsuji, T. Shitara, and M. Ueda, Out-of-time-order fluctuation-dissipation theorem, Phys. Rev. E 97, 012101 (2018).

[27] J.-T. Hsiang and B. L. Hu, Atom-field interaction: From vacuum fluctuations to quantum radiation and quantum dissipation or radiation reaction, Physics 1, 430 (2019).

[28] J.-T. Hsiang and B. L. Hu, preceding paper, Nonequilibrium nonlinear open quantum systems I: Functional perturbative analysis of a weakly anharmonic oscillator, Phys. Rev. D 101, 125002 (2020).

[29] L. C. Lapas, I. V. L. Costa, M. H. Vainstein, and F. A. Oliveira, Entropy, non-ergodicity and non-Gaussian behaviour in ballistic transport, Europhys. Lett. 77, 37004 (2007).

[30] B. L. Hu, J. P. Paz, and Y. Zhang, Quantum Brownian motion in a general environment II. Nonlinear coupling and perturbative approach, Phys. Rev. D 47, 1576 (1993).

[31] J. S. Schwinger, Brownian motion of a quantum oscillator, J. Math. Phys. (N.Y.) 2, 407 (1961); L. Keldysh, Diagram technique for nonequilibrium processes, Zh. Eksp. Teor. Fiz. 47, 1515 (1964) [JETP 20, 1018 (1964)], http://www.jetp.ac .ru/cgi-bin/e/index/e/20/4/p1018?a=list; K.-C. Chou, Z.-B. $\mathrm{Su}$, B.-L. Hao, and L. Yu, Equilibrium and non-equilibrium formalisms made unified, Phys. Rep. 118, 1 (1985).

[32] C. Moreno and J.-D. Urbina, Strong coupling and nonMarkovian effects in the statistical notion of temperature, Phys. Rev. E 99, 062135 (2019).

[33] J. D. Jackson, Classical Electrodynamics, 2nd ed. (Wiley, New York, 1975). 